Article

\title{
Human cytomegalovirus essential tegument protein pp150 is amenable to targeting by se- quence specific peptides and ELP-conjugates
}

Dipanwita Mitra ${ }^{1}$, Mohammad H. Hasan ${ }^{1}$, Gene L. Bidwell2,3,4, and Ritesh Tandon ${ }^{1,5,6 *}$

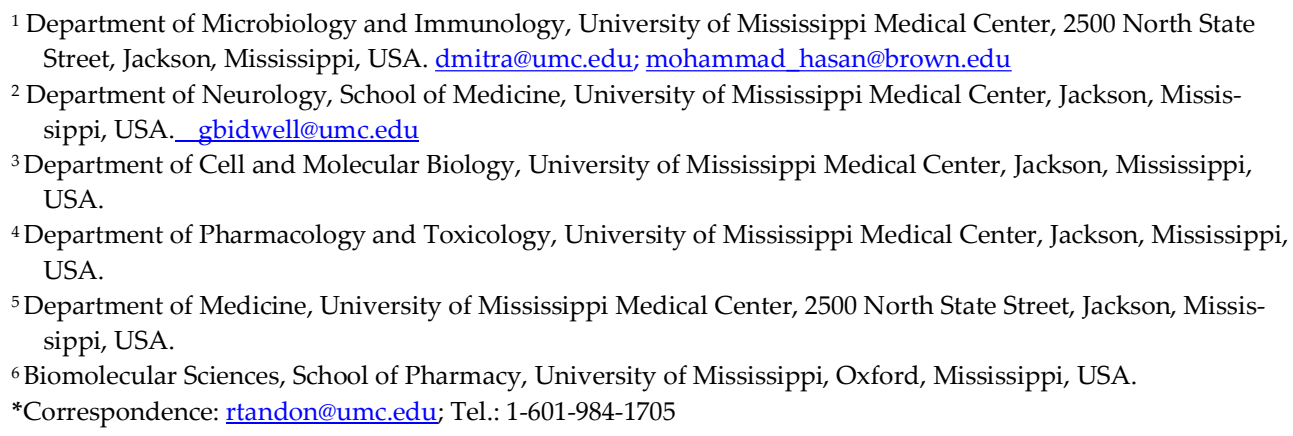

Abstract: Human cytomegalovirus (HCMV) tegument protein pp150 is essential for the completion of final steps in virion maturation. Earlier studies indicated that three pp150nt (N terminal one-third of pp150) conformers cluster on each triplex (Tri1, Tri2A and Tri2B) and extend towards small capsid proteins atop nearby major capsid proteins forming a net-like layer of tegument densities that enmesh and stabilize HCMV capsids. Based on this atomic detail, we designed several peptides targeting pp150nt. Our data show significant reduction in virus growth upon treatment with one of these peptides (pep-CR2) with an IC50 of $1.33 \mu \mathrm{M}$. Based on 3D modeling, pep-CR2 specifically interferes with the pp150-capsid binding interface. Cells pre-treated with pep-CR2 and infected with HCMV sequester pp150 in the nucleus indicating a mechanistic disruption of pp150 loading onto capsids and subsequent nuclear egress. To enhance the in-vivo inhibitory potential and bioavailability of pep-CR2, we conjugated it with a carrier molecule (elastin like polypeptide (ELP)). The ELP-pep-CR2 conjugate was expressed in E.coli and purified. Upon treatment with ELP-pep-CR2, HCMV showed significant titer reductions with no significant impact on cell viability. These results indicate that CR2 of pp150 is amenable to targeting by a peptide inhibitor and can be developed into an effective antiviral.

Keywords: CMV; herpesviruses; peptide therapy; tegument; nuclear egress

\section{Introduction}

Human Cytomegalovirus (HCMV), a betaherpesvirus, infects the majority of the world's population, but acute disease is manifested only in a small proportion of infected individuals. Primary infection or reactivation of latent virus can cause life threatening complications in immunocompromised individuals such as AIDS patients and transplant recipients. HCMV is also the leading cause of congenital infections and is associated with a multitude of cardiovascular diseases. Currently, there are no commercially available 
vaccines for HCMV infection. Antivirals are available; however, adverse side effects and drug resistance are a growing concern [1-4] .

HCMV has a prototypical herpesvirus virion (infectious virus particle) that consists of an external membranous envelope, a proteinaceous layer called the tegument and an icosahedral capsid that contains a compressed large $(>240 \mathrm{~kb})$ double stranded DNA genome. Like all herpesviruses, HCMV maturation occurs in two distinct phases, primary and secondary maturation. Primary maturation begins in the host cell nucleus where viral genome replication, capsid assembly and encapsidation takes place. After assembly, the nucleocapsids (NC) migrate from the nucleus to the cytoplasm. During this nuclear egress, the nucleocapsid first undergoes a primary envelopment at the inner nuclear membrane, traverses through the nuclear envelope, followed by de-envelopment at the outer nuclear membrane, and finally reaches the cytoplasm where it accumulates within a ring-shaped perinuclear structure known as the cytoplasmic virus assembly compartment (vAC) where the secondary or final steps of virion maturation occur [5-10].

The virion maturation process is characterized by numerous virus-virus, host-host and host-virus interactions, and the tegument proteins play critical roles during this process $[4,9,10]$. The tegument proteins are also active during viral entry as they are released into the cells along with viral capsids and aid in viral gene expression, virus replication and evasion of host immune responses [11,12]. During late stages of infection, tegument proteins accumulate to high levels in the vAC and contribute to virion maturation and egress [9-11]. Thus, tegument proteins offer important targets for the development of antiviral therapy against HCMV infection.

The HCMV UL32 gene encodes a prominent betaherpesvirus conserved virion tegument protein, pp150 (basic phosphoprotein/ppUL32), which is intially expressed in the nucleus but accumulates within the vAC during late stages and supports the final steps in virion maturation $[13,14]$. To date, several studies have been conducted to understand the structure and function of pp150 [13-23]. It is well established that pp150 controls cytoplasmic events during virion maturation [14,16,17]. The principal role of pp150 is to stabilize and retain the nucleocapsid organization throughout the final or secondary envelopment inside the $\mathrm{VAC}[9,13,14]$. The cryoEM reconstructions of HCMV virion show that pp150 is arranged in upper and lower helix bundles which is joined by a central helix $[18,20,24,25]$. The HCMV capsid is an ensemble of 60 asymmetric units, with each of these units containing i) Sixteen copies of the major capsid protein (MCP), which exists in penton and hexon capsomers, ii) Sixteen copies of small capsid proteins (SCP) that sit atop each $\mathrm{MCP}$, iii) Five and one-third heterotrimeric triplexes $(\mathrm{Ta}, \mathrm{Tb}, \mathrm{Tc}, \mathrm{Td}$, $\mathrm{Te}$ and $\mathrm{Tf}$ respectively) composed of the triplex monomer protein Tri1 (minor capsid binding protein), which is coupled with triplex proteins Tri2A and Tri2B (also known as minor capsid proteins) and iv) Sixteen copies of the pp150 molecules $[4,20,24]$. Three pp150 proteins cluster above each triplex and extend towards the three SCPs atop nearby MCPs 
forming a net-like layer of tegument densities that enmesh HCMV capsids [24]. The atomic model construct for the N-terminal third of pp150 (pp150nt; residues 1 to 285) suggests that $\mathrm{N}$-terminal residues 1 to 275 alone are sufficient for pp150-capsid binding [24]. Several conserved regions in the $\mathrm{N}$ terminal 275 residues have been identified, including a 27 amino acid cysteine tetrad region which is conserved across all primate CMVs and two betaherpesvirus conserved regions (CR1 and CR2) [13-15]. The cysteine tetrads and CR1 lie in pp150nt's upper helix bundle whereas CR2 lies in the lower helix bundle (Figure 1). pp150nt-capsid interactions occur in both upper and lower helix bundles and stabilizes the nucleocapsid for the production of infectious HCMV virion $[20,24]$.

We targeted the conserved pp150nt regions with sequence specific-peptides developed with the goal of developing these peptides into highly effective antivirals. The atomic details of pp150nt structure and its binding interface with capsid proteins (Figure 1A and 1B) guided the design of peptides targeting CR1, CR2 and cysteine tetrad regions (Table 1; Figure 2). The data show that at least one of these peptides (pep-CR2) is effective in inhibiting CMV growth in cell culture. Peptide therapeutics are a promising new strategy for targeted therapy; however, peptides can rapidly degrade in an in-vivo environment and also suffer from poor pharmacokinetic parameters as well as low tissue and cell membrane permeability [26-28]. To overcome these limitations, we used elastin like polypeptide (ELP) as a carrier molecule to enhance the inhibitory potential and in-vivo bioavailability of pep-CR2 [26]. ELPs are biopolymers derived from mammalian elastin and are composed of a short repeating peptide motif of valine-proline-glycine-X-glycine (VPGXG), where $X=$ a guest amino acid, that can be engineered to produce viscoelastic properties similar to elastin [29]. ELPs have been well studied for drug delivery and tissue engineering. They are biocompatible, resorbable, non-immunogenic and have a lower critical solution temperature phase transition behavior which makes them extensively useful for drug delivery in different manifestations such as soluble macromolecular carriers, self-assembled nanoparticles, cross-linked microparticles, or thermally coacervated depots [26,30-36]. ELP-pep-CR2 conjugate showed HCMV inhibitory potential similar to the unconjugated pep-CR2 without any significant impact on cell viability. Overall, the results in this study indicate that CR2 of pp150 is amenable to targeting by a peptide inhibitor, which can be developed into an effective antiviral. 


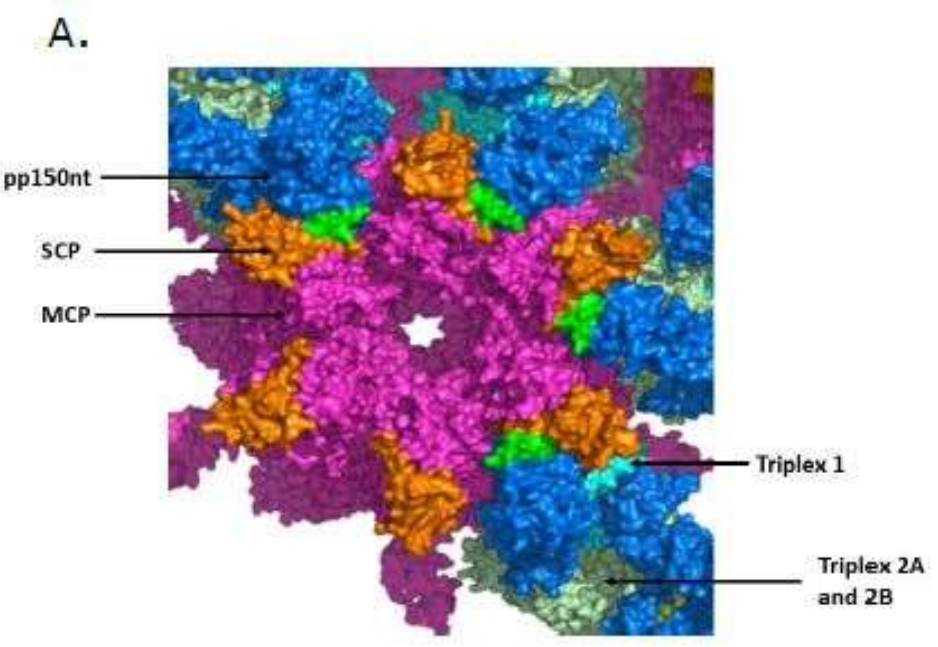

B.

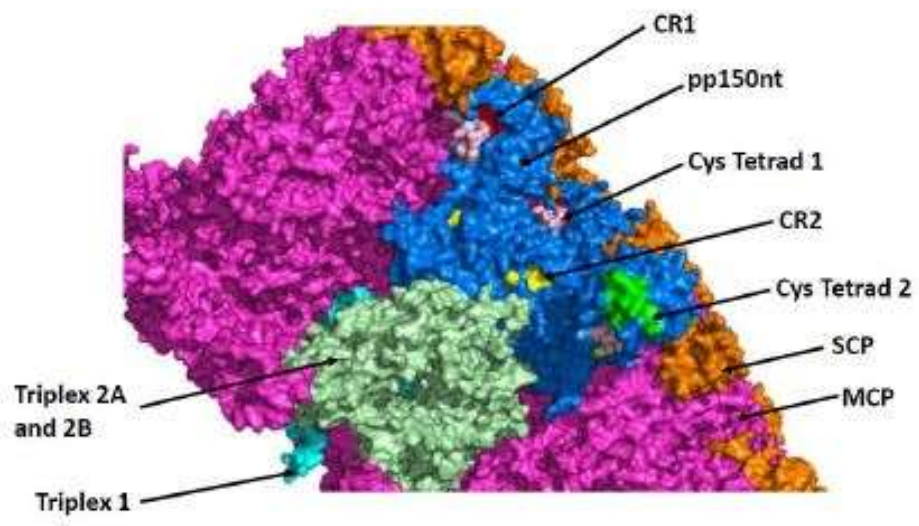

Figure 1. A) Illustration of pp150 structure and its binding interface with capsid proteins in an HCMV virion. MCP (major capsid proteins) makes up pentons and hexons. Triplexes (also known as minor capsid binding protein) are heterotrimers composed of Tri1 and a Tri2A-Tri2B heterodimer. SCP (small capsid protein) binds to MCP. pp150 tegument protein clusters above triplexes and extends towards SCP atop nearby MCP forming a net-like layer of tegument densities. Magenta-MCP; Orange-SCP; Blue-pp150; Cyan-Triplex 1; Pale green-Triplex 2A and 2B. B) Close-up view showing conserved regions in pp150. Magenta-MCP; Orange-SCP; Bluepp150; Cyan-Triplex 1; Pale green-Triplex 2A and 2B; Red-pp150 conserved region 1 (CR1); Yellow- pp150 conserved region 2 (CR2); Pink-Cysteine tetrad 1; Green-Cysteine tetrad 2. Created in PyMOL software (Schrödinger, LLC) using RSCB PDB ID: 5VKU [24].

Table 1. Amino acid sequence of test and control peptides ${ }^{1}$

\begin{tabular}{ll}
\hline Peptide & \multicolumn{1}{c}{ Sequence } \\
\hline Pep-control & DYKDDDDK (Flag Sequence) Control \\
Pep-CR1 & LFNELMLWL (Resembles CR1) \\
Pep-CR2 & NKLVYTGRL (Resembles CR2) \\
Pep-CysTetrad1 & KCLARIQERCK (Resembles Cysteine Tetrad) \\
Pep-CysTetrad2 & MCLSFDSNYCR (Resembles Cysteine Tetrad) \\
\hline${ }^{1}$ All peptides were synthesized with a N-terminal myristoylation by GenScript, USA.
\end{tabular}




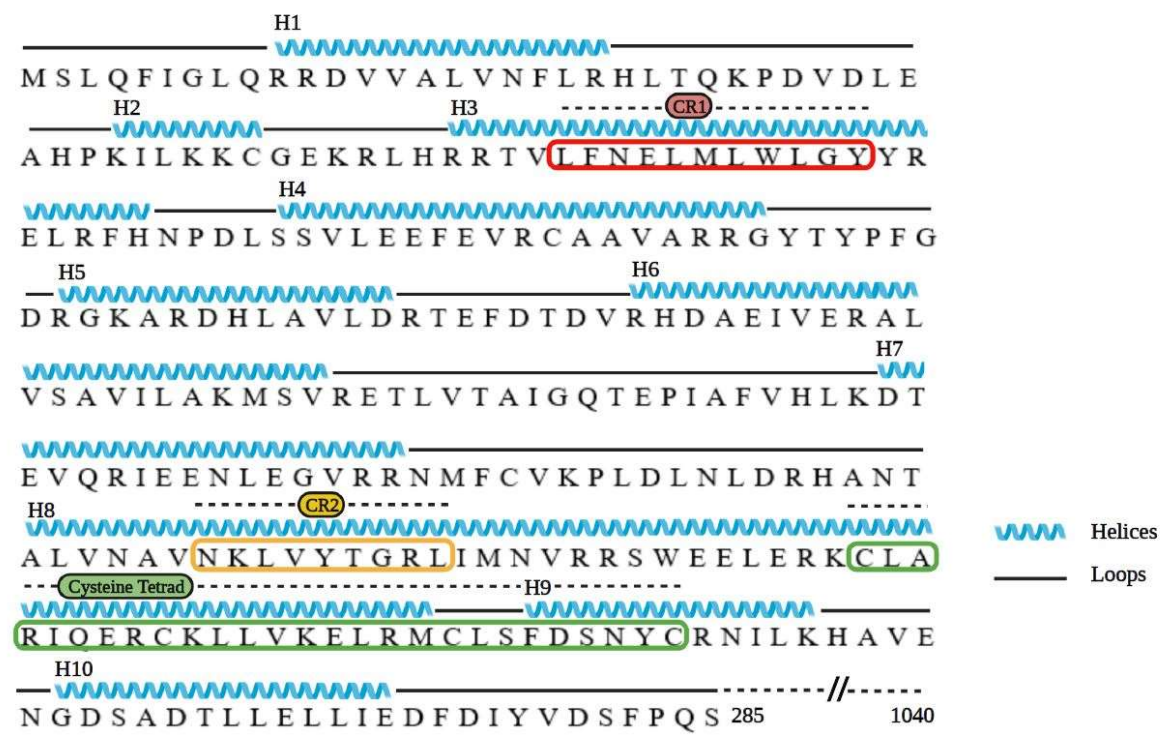

Figure 2. Amino acid sequence and predicted secondary structure of pp150nt. Black lines represent loops, blue lines represent helices, CR1 is boxed in red, CR2 boxed in yellow and cysteine tetrad boxed in green. Created using PyMOL software (Schrödinger, LLC) using RSCB PDB ID: 5 VKU [24].

\section{Materials and Methods}

Preparation of peptides: Peptides were designed with similarity to amino acid sequences of the conserved regions in pp150nt (Table 1). All peptides were modified to include a Nterminal myristoyl group for better cell penetration and synthesized by GenScript, USA to $>85 \%$ purity. The lyophilized peptides were resuspended in $100 \%$ dimethyl sulfoxide (DMSO) before use; however, the working concentration of the peptides included less than $0.1 \%$ DMSO.

Cells: Human foreskin fibroblasts (HFF) were cultured in Dulbecco's modified Eagle's medium (DMEM; Corning, Manassas, VA; catalog\# 10-013-CM) containing 10\% fetal bovine serum (Gibco, Life Technologies, Grand Island, NY, catalog\# 10437-028), 4.5 g/ml glucose, $2 \mathrm{mM}$ L-glutamine, $1 \mathrm{mM}$ sodium pyruvate, and $100 \mathrm{U} / \mathrm{ml}$ penicillin-streptomycin (Corning, Manassas, VA; catalog\# 30-002-CI) at $37^{\circ} \mathrm{C}$ with $5 \% \mathrm{CO} 2$.

Virus: HCMV (TowneBAC and BAD32 strain) was grown on HFF cells. Virus stock was prepared in $3 \mathrm{X}$ autoclaved milk, sonicated 3 times for 10 seconds with 30 seconds gap, and stored at $-80{ }^{\circ} \mathrm{C}$. $3 \mathrm{X}$ autoclaved milk is prepared from Carnation (Nestle) instant nonfat dry milk powder. $10 \%$ milk was prepared in nano pure water, $\mathrm{pH}$ was adjusted to 7.0 and was autoclaved for three times.

Virus infection: During infection, media was removed from the wells of cell culture plates and appropriately diluted virus stock was absorbed onto the cells in DMEM without serum. Cells were incubated for 1 hour with gentle shaking every 10 mins followed by washing 3X with serum free DMEM. Fresh complete medium was added and cells were incubated until the end point. 
Virus titers: Infected or mock-infected samples were harvested within the medium at the designated time points post infection and stored at $-80^{\circ} \mathrm{C}$ before titration. On the day of titration, harvested samples were sonicated three times for 10 seconds each with 30 seconds gap. Monolayers of HFF were grown in twelve well tissue culture plates, and serial dilutions of sonicated samples were absorbed onto them for 1 hour, followed by $3 \mathrm{X}$ washing with serum free DMEM. Fresh DMEM containing 10\% FBS was added, and cells were incubated for 9 to 10 days post infection (dpi). At the end point, DMEM was removed and cells were washed $2 X$ with PBS. Infected monolayers were fixed in $100 \%$ methanol for 5 mins and immediately stained with Giemsa stain, modified (Sigma-Aldrich, MilliporeSigma, US catalog\#GS1L) for 15 mins. Plates were finally washed with tap water, air dried, and plaques with clear zone were quantified.

Microscopy: Samples were prepared using established protocols for fluorescence microscopy. Briefly, HFF cells were grown on coverslip inserts in 24-well tissue culture plates. At the end point, cells were fixed in 3.7\% formaldehyde for $10 \mathrm{~min}$ and were incubated in $50 \mathrm{mM} \mathrm{NH}_{4} \mathrm{Cl}$ in PBS for 10 mins to reduce auto-fluorescence. Followed by 2X PBS wash, cells were incubated in 0.5\% Triton X-100 for 20 mins for permeabilization and washed with PBS. Finally cells were incubated in Hoechst solution (ThermoFisher Scientific, USA, Catalog\# 33342) in PBS (1:3000) for 10 mins to stain the nucleus, followed by PBS wash. Coverslips were retrieved from the wells and were mounted on glass slides with a drop of mounting medium $(2.5 \%$ DABCO in Fluoromont G) and air dried for two hours before imaging. Images were acquired on an EVOS-FL epifluorescent microscope (ThermoFisher Scientific, USA).

Cell viability: HFF cells were plated in 24 well tissue culture plates and grown to confluency. Cells were pretreated for 1 hour with appropriate concentration of controls and test peptides and then infected with HCMV at a multiplicity of infection (MOI) of 3.0 or mock-infected. At 3 dpi, cells received fresh complete DMEM containing fresh peptides or controls. Media was removed at $5 \mathrm{dpi}$, and cells were harvested by trypsinization. Cell viability was determined using trypan blue exclusion using a TC20 automated cell counter (BioRad Laboratories, Hercules, CA) following the manufacturer's protocol.

Generation of ELP-peptide construct: A synthetic DNA casette endcoding the pep-CR2 sequence was synthesized with codons that were optimized for expression in E. coli (Life Technologies). The casette was inserted into the pET26b expression vector between NdeI and BamHI restriction sites, with an SfiI site at the N-terminus of the pep-CR2 coding sequence. DNA containing the ELP coding sequence was excised from pUC19-ELP and cloned into the SfiI site, generating an in-frame fusion of ELP and pep-CR2 (Figure S1). The ELP sequence contained 160 VPGxG repeats in which the $x$ residue was $V$, G, or A in a 1:7:8 ratio. All constructs were confirmed by DNA sequencing [37].

Purification of ELP-pep-CR2: The pET25b+ vector containing the ELP-pep-CR2 coding sequence was transformed into E. coli BLR(DE3) and selected on ampicillin agar plates. Liquid cultures (10 mL Terrific Broth (TB Dry, MP Biomedicals)) were innoculated and 
grown overnight, then used to innoculate $500 \mathrm{~mL}$ cultures that were grown for 16 - 20 hours in 2L shaker flasks. The pET system can produce low-level recombinant protein expression even without induction. Bacteria were harvested by centrifugation, lysed by sonication, and nucleic acids were precipitated with polyethyleneimine (PEI) and removed by centrifugation [38]. The salt concentration of the soluble lysate was raised to approximately $200 \mathrm{mg} / \mathrm{mL}$ by addition of $\mathrm{NaCl}$, and the solution was heated at $50^{\circ} \mathrm{C}$ until the ELP-pep-CR2 precipitated (as judged by the formation of a visibly cloudy solution). The precipitated ELP-pep-CR2 was collected by centrifugation, and the protein pellet was re-dissolved in cold PBS and centrifuged at $4^{\circ} \mathrm{C}$ to remove any un-dissolved precipitate. This heat cycling process was repeated $3-5$ times until purified protein was obtained. Purity was assessed by SDS-PAGE [37] (Figure S2), and the concentration of ELP-pep-CR2 was determined spectrophotometrically by measuring absorbance at $280 \mathrm{~nm}$ and utilizing the theoretical extinction coefficient of $6990 \mathrm{M}^{-1} \mathrm{~cm}^{-1}$, which was calculated from the protein's primary sequence using the Expasy Protparam tool.

Statistics: Student's t-tests were conducted in Graphpad Prism comparing the means of different groups (GraphPad Prism version 8.0.0, GraphPad Software, San Diego, California USA, www.graphpad.com). Standard error of mean was plotted as error bars. A p value of $<0.05$ was considered significant. An asterisk $\left(^{*}\right)$ indicates significant inhibition compared to wild-type.

\section{Results}

\subsection{Inhibition of virus growth upon pep-CR2 treatment}

First, we sought to establish the inhibitory efficiency of the peptides. Primary human foreskin fibroblasts (HFF) were pretreated with pep-CR1, pep-CR2, pep-CysTetrad1, pepCysTetrad2, pep-control and DMSO at $10 \mu \mathrm{M}$ concentration for 1 hour before infecting them with HCMV at an MOI of 3.0. Peptide concentrations were maintained in the infected cell culture. Cells were harvested at 5 days post infection (dpi), and virus plaque forming units were enumerated by plating of serial dilutions on fibroblasts. The titers indicated $>10-$ fold reduction in virus growth in pep-CR2 treated cells but not when treated with pepCR1, pep-CysTetrad1, pep-CysTetrad2, pep-control and DMSO (Figure 3). Next, to determine the half maximal inhibitory concentration $\left(\mathrm{IC}_{50}\right)$ of pep-CR2, HFFs were pretreated with pep-CR2 (with a concentration range of $1 \mu \mathrm{M}-20 \mu \mathrm{M}$ ), pep-control and ganciclovir (GCV; as an inhibitory control). Cells were then infected with HCMV at an MOI of 0.1 , and virus yield was measured at $10 \mathrm{dpi}$ by enumerating the plaque forming units. The percent inhibition of HCMV by pep-CR2 was plotted against the treatment concentration (Figure $4 \mathrm{~A}$ ), and the $\mathrm{IC}_{50}$ was calculated to be $1.33 \mu \mathrm{M}$ with a $95 \%$ confidence interval of 1.182 to $1.474 \mu \mathrm{M}$. Compared to the pep-control, pep-CR2 showed $90 \%$ inhibition of virus titers at $10 \mu \mathrm{M}$ concentration (Figure $4 \mathrm{~B}$ ), confirming previous results at higher MOI (Figure 3). To test the cytotoxicity of pep-CR2, a cell viability assay was performed on pep-CR2 (10uM) treated infected and uninfected cells along with appropriate controls. Cell viability was similar across treatment groups in this assay (Figure S3A). Furthermore, pep-CR2 treated infected cells had significantly higher cell 
viability compared to the control groups, indicating that pep-CR2 efficiently protected the HCMV infected cells from virus induced lytic death (Figure S3B). Overall, the data from these experiments indicate significant reduction in virus growth upon pep-CR2 treatments without impacting cell viability.

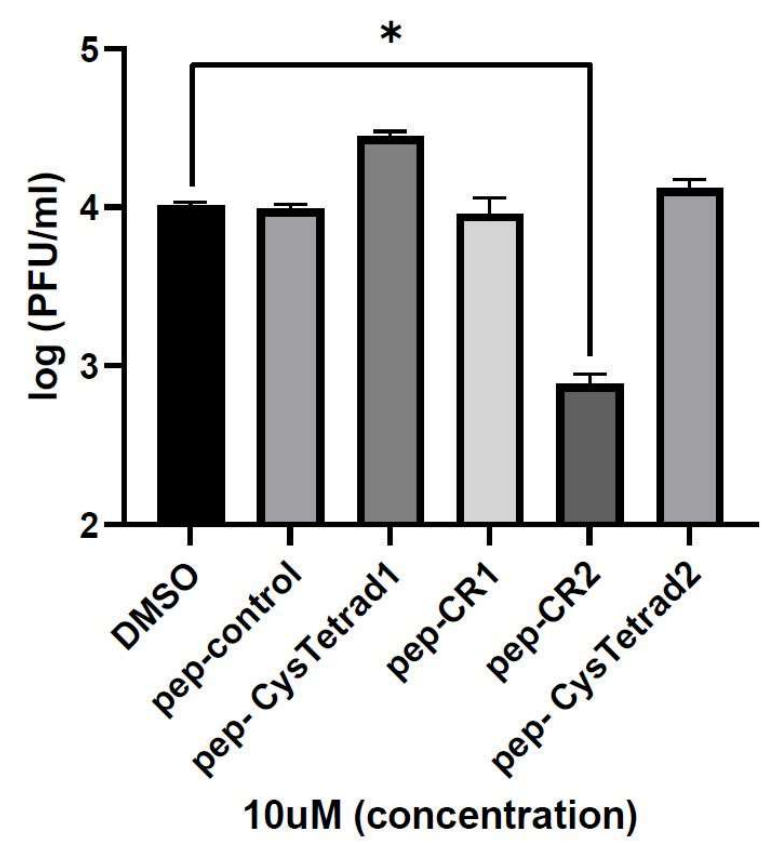

Figure 3. Inhibition of virus growth upon pep-CR2 treatment. Cells (human foreskin fibroblasts (HFF)) were pretreated with $10 \mu \mathrm{M}$ concentration of control (DMSO), pep-control, pep-CysTetrad1, pep-CysTetrad2, pep-CR1 and pep-CR2 and infected with HCMV at an MOI of 3.0. Cells were harvested at $5 \mathrm{dpi}$ (days post infection) and virus titers were assessed on HFFs. Data was analyzed by student's t-tests comparing the means of DMSO control and the peptidetreated group. Standard error of mean was plotted as error bars. A p value of $<0.05$ was considered significant. An asterisk $\left(^{*}\right)$ indicates significant inhibition compared to wild-type.

\section{Pep-CR2 treatment sequesters pp150 in the nucleus of infected cells}

To study the localization of pp150 in pep-CR2 and pep-control treated infected cells, HFFs were pretreated with $10 \mu \mathrm{M}$ concentration of pep-CR2 and pep-control for 1 hour and then infected with the BAD32 strain of HCMV (MOI 3.0), a strain in which the late protein pp150 is GFP tagged [22]. Cells were fixed and imaged by epifluorescent microscopy at 4 dpi. These localization studies showed that pep-CR2 treated cells contain diffused GFP signal in the cytoplasm of infected cells in contrast to a solid juxtanuclear sphere of GFP which corresponds to vAC in the pep-control treated cells (Figure 5). The intensity of GFP signal was much higher in the nucleus of pep-CR2 treated cells compared to the nucleus of the pep-control treated cells. These results indicate that pep-CR2 may be compromising virion maturation by sequestering pp150 in the nucleus of infected cells and interfering with the organization of the vAC. 

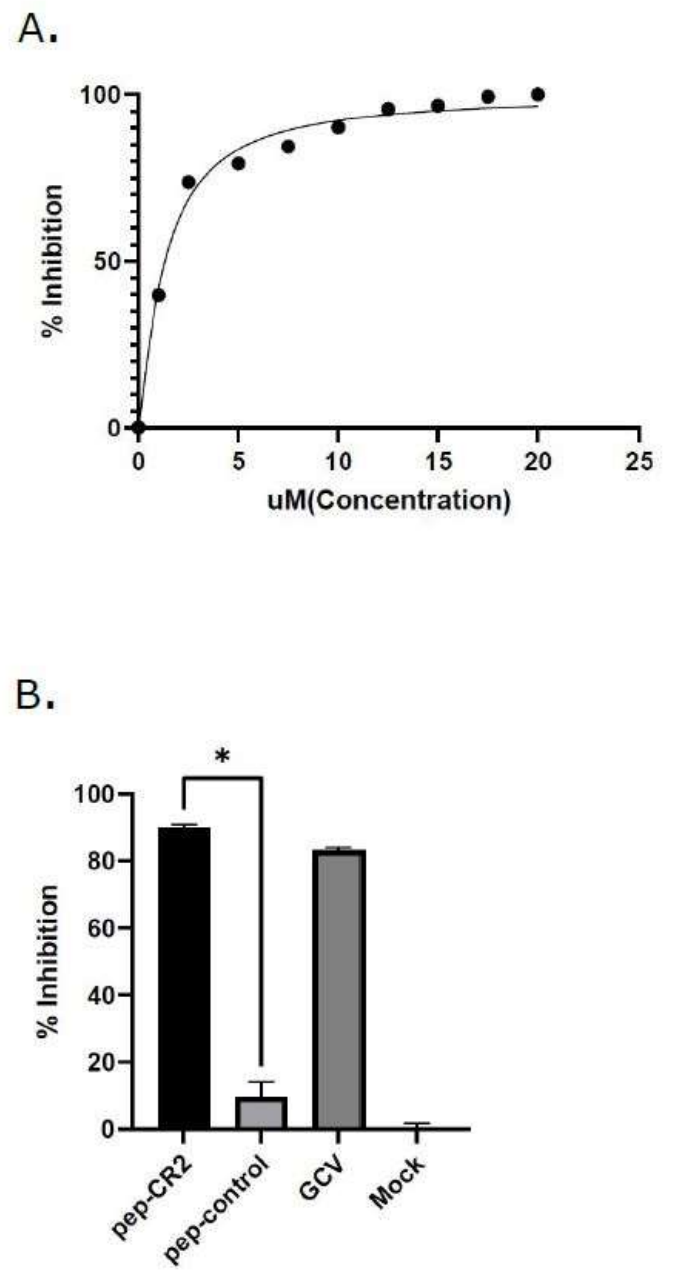

Figure 4. A) Determining the $\mathrm{IC}_{50}$ (half maximal inhibitory concentration) of pep-CR2. HFFs were pretreated for 1 hour with different concentrations of pep-CR2 $(1 \mu \mathrm{M}, 2.5 \mu \mathrm{M}, 5 \mu \mathrm{M}, 7.5 \mu \mathrm{M}, 10 \mu \mathrm{M}$, $12.5 \mu \mathrm{M}, 15 \mu \mathrm{M}, 17.5 \mu \mathrm{M}$ and $20 \mu \mathrm{M}$ ), pep-control, ganciclovir (GCV) or mock and then infected with HCMV at a low MOI of 0.1. Cells were fixed at $10 \mathrm{dpi}$, and virus yield was measured by counting the number of plaques. The percentage inhibition of pep-CR2 is plotted against concentration range of $1 \mu \mathrm{M}-20 \mu \mathrm{M}$. The IC 50 of pep-CR2 was calculated to be at $1.33 \mu \mathrm{M}$ with a $95 \%$ confidence interval of 1.182 to $1.474 \mu \mathrm{M}$. B) Comparing inhibition of virus growth by pep-CR2, pep-control, GCV and no treatment control (Mock) at $10 \mu \mathrm{M}$ concentration. Pep-CR2 treated cells showed $\sim 90 \%$ inhibition in virus growth compared to the controls. Data was analyzed by student's t-tests comparing the means of control and treatment groups. Standard error of mean was plotted as error bars. A p value of $<0.05$ was considered significant. An asterisk $\left(^{*}\right)$ indicates significant inhibition compared to wildtype. 


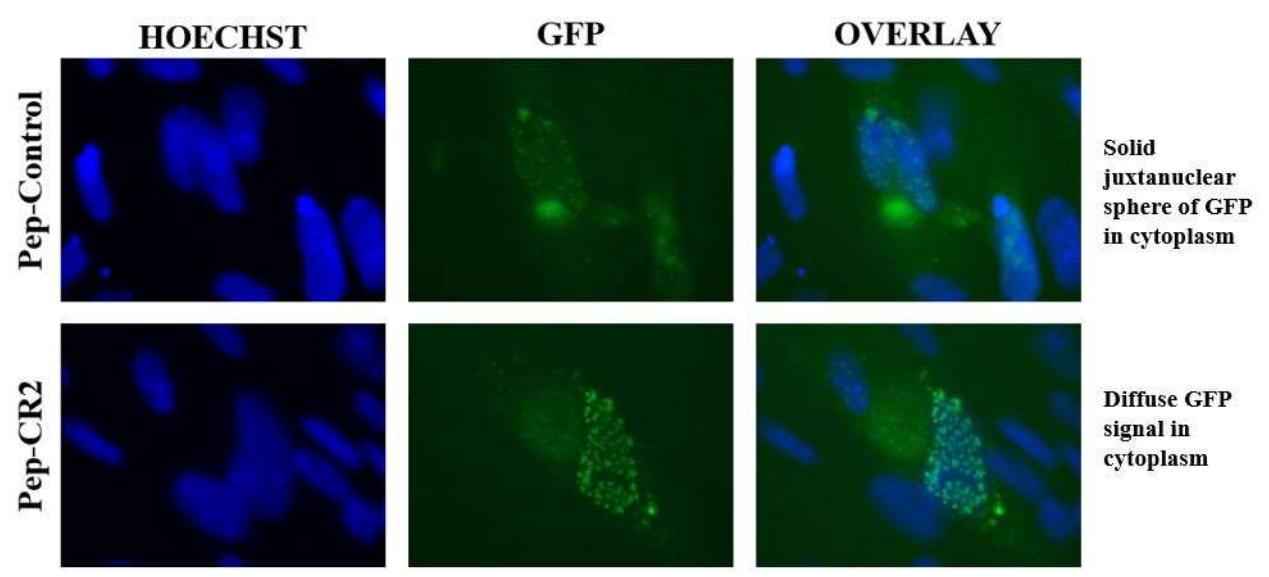

Figure 5. Epifluorescent imaging indicating pp150 localization in the nucleus and the cytoplasm of pep-CR2 and pep-control treated cells. ells were pretreated with pep-control and pep-CR2 at $10 \mu \mathrm{M}$ concentration for 1 hour before infecting them with HCMV (BAD32 strain where pp150 is GFP tagged) at an MOI of 3.0. Cells were fixed and imagCed under an epifluorescent microscope at $4 \mathrm{dpi}$. Hoechst stains the nucleus. Results indicate that pep-CR2 may be compromising virion maturation by sequestering pp150 in the nucleus of infected cells and interfering with the organization of the virus assembly compartment (vAC).

\section{ELP-pep-CR2 shows similar inhibitory potential to pep-CR2 in HCMV infected HFFs}

The inhibitory potential of the protein biopolymer - delivered peptide, ELP-pep-CR2, was determined in comparison to the inhibitory potential of the free pep-CR2 peptide. HFF cells were pretreated with ELP-pep-CR2 with a concentration range of $8.05 \mu \mathrm{M}$ to $161 \mu \mathrm{M}$ and appropriate controls for 1 hour and then infected with HCMV at a low MOI of 0.1. Cells were fixed at $10 \mathrm{dpi}$, and virus yield was measured by counting the number of plaques. The half maximal inhibitory concentration ( $\left.\mathrm{IC}_{50}\right)$ of ELP-pep-CR2 was calculated to be at $21.33 \mu \mathrm{M}$ with $95 \%$ confidence interval of 18.93 to $23.63 \mu \mathrm{M}$ (Figure 6A). Results show significant inhibition of virus growth in infected cells upon treatment with ELP-pepCR2 compared to mock (zero concentration) (Figure 6A). However, comparing the IC 50 of pep-CR2 (Figure 4) and ELP-pep-CR2, indicate that ELP-delivered peptide is approximately 16X less potent than the free peptide.

Interestingly, the ELP-control itself also showed some inhibition of virus in infected cells (Figure 6B). To analyze whether the inhibition by ELP-control is different from specific inhibition by ELP-pep-CR2, the half maximal inhibitory concentration (IC50) of ELP-control was calculated using an 8.93-178.6 $\mu \mathrm{M}$ concentration range. The IC 50 of ELP-control was calculated to be at $98.04 \mu \mathrm{M}$ with a $95 \%$ confidence interval of 87.69 to $107.4 \mu \mathrm{M}$ (Figure $6 \mathrm{~B})$. The results indicate that the IC 50 of ELP-control is significantly higher than the IC 50 of ELP-pep-CR2 $(21.23 \mu \mathrm{M})$ and the inhibitory effect of the ELP-pep-CR2 treatment group is significantly greater compared to the ELP-control group. To further assess the impact of ELP-control on virus spread, plaque sizes were calculated and compared between ELPcontrol vs. ELP-pep-CR2 treated groups. ELP-pep-CR2 treated infected cells showed significantly smaller plaque sizes compared to the ELP-control group, indicating reduction in 
virus spread to the adjacent cells in a focus, hence inhibition of virus growth (Figure 7A, 7B).

Next, to rule out any cytotoxic effects of ELP-pep-CR2, cell viability assay was performed on ELP-pep-CR2 treated infected and uninfected HFFs along with ELP-control and GCV as appropriate controls. Cell viability was not affected at the treated concentration of ELPpep-CR2 in uninfected cells (Figure S4A). Also, ELP-pep-CR2 treated infected cells had higher cell viability compared to control groups indicating that ELP-pep-CR2 efficiently protected the HCMV infected cells from virus induced lytic death (Figure S4B). Overall, these results indicate that ELP-pep-CR2 show similar inhibitory potential compared to pep-CR2 in HCMV infected cells.
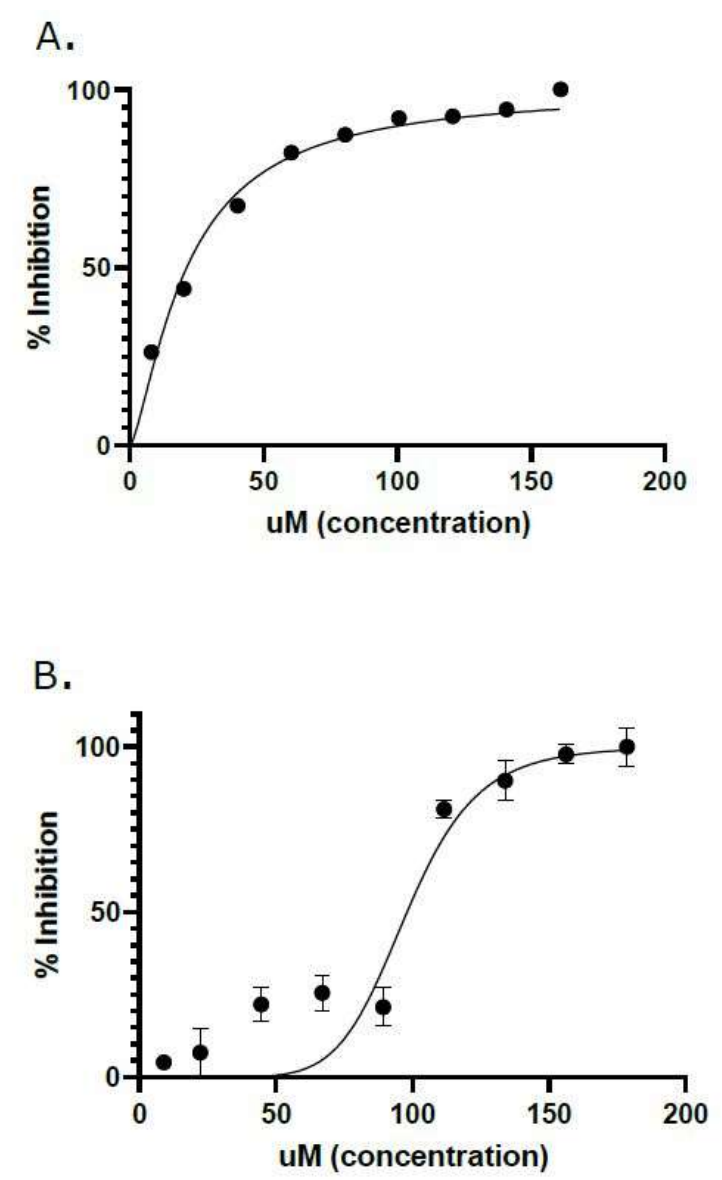

Figure 6. Assessing the inhibitory potential of ELP-pep-CR2 in HFFs. A) Determination of the IC 50 of ELP-pep-CR2. HFFs were pretreated for 1 hour with increasing concentrations of ELP-pep-CR2 (8.05 $\mu \mathrm{M}, 20.125 \mu \mathrm{M}, 40.25 \mu \mathrm{M}, 60.375 \mu \mathrm{M}, 80.5 \mu \mathrm{M}, 100.625 \mu \mathrm{M}, 120.75 \mu \mathrm{M}, 140.875 \mu \mathrm{M}$ and 161 $\mu \mathrm{M})$, with appropriate control groups, and then infected with HCMV at a low MOI of 0.1. Cells were fixed at $10 \mathrm{dpi}$ and virus yield was measured by counting the number of plaques. The $\%$ inhibition of ELP-pep-CR2 is plotted against concentration range of 8.05-161 $\mu \mathrm{M}$. The IC50 of ELP-pep-CR2 was calculated to be at $21.33 \mu \mathrm{M}$ with a $95 \%$ confidence interval of 18.93 to $23.63 \mu \mathrm{M}$. B) Determining the IC 50 of ELP-control. HFFs were pretreated for 1 hour with increasing concentrations of ELPcontrol $(8.93 \mu \mathrm{M}, 22.325 \mu \mathrm{M}, 44.65 \mu \mathrm{M}, 66.975 \mu \mathrm{M}, 89.3 \mu \mathrm{M}, 111.625 \mu \mathrm{M}, 133.95 \mu \mathrm{M}, 156.275 \mu \mathrm{M}$ and 
$178.6 \mu \mathrm{M}$ ) and approprotiate control groups, and then infected with HCMV at a low MOI of 0.1. Cells were fixed at $10 \mathrm{dpi}$ and virus yield was measured by counting the number of plaques. The \% inhibition of ELP-control is plotted against concentration range of 8.93-178.6 $\mu \mathrm{M}$. The IC50 of ELPcontrol was calculated to be at $98.04 \mu \mathrm{M}$ with a $95 \%$ cnfidence interval of 87.69 to $104.7 \mu \mathrm{M}$.

A.

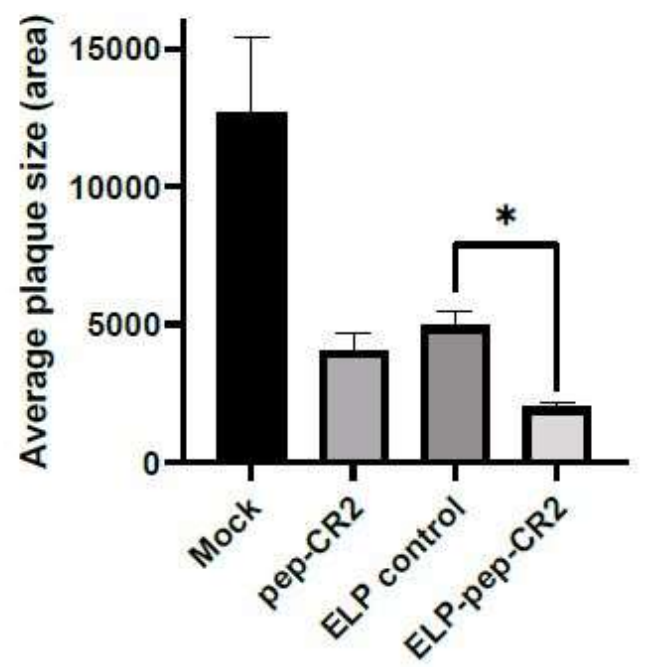

B.

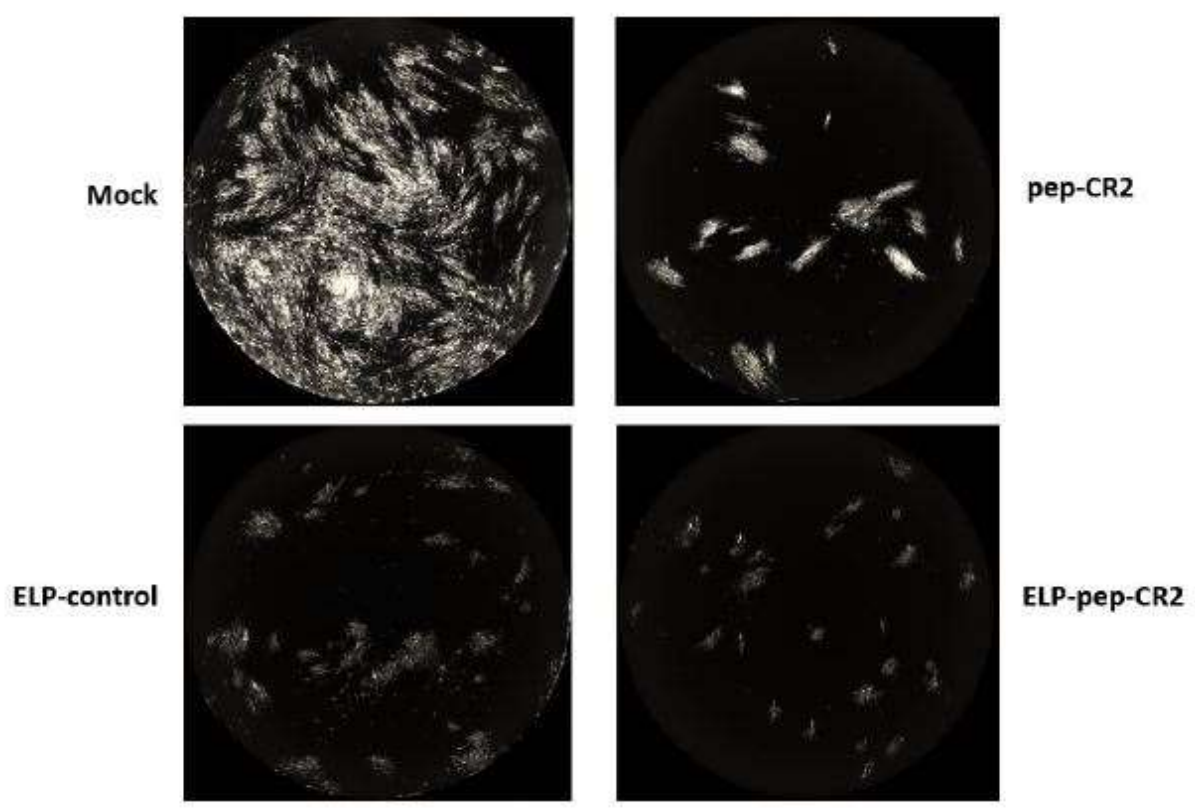

Figure 7. A) Plaque size comparison between ELP-pep-CR2, ELP-control and pep-CR2 treated cells. Cells were pretreated with ELP-pep-CR2 $(80 \mu \mathrm{M})$, ELP-control $(89.3 \mu \mathrm{M})$ and pep-CR2 $(10 \mu \mathrm{M})$ for 1 hour before infecting them with HCMV at a low MOI of 0.1. Cells were fixed at $10 \mathrm{dpi}$ and virus yield was measured by counting the number of plaques. Individual plaque sizes were measured by counting the area of the plaques in ImageJ software (National Institutes of Health, Bethesda, MD, USA). Data was analyzed by student's t-tests comparing the means of the test and the control group. 
Standard error of mean was plotted as error bars. A p value of $<0.05$ was considered significant. An asterisk $\left(^{*}\right)$ indicates significant inhibition compared to wild-type. B) GFP+ images showing plaque size comparison in ELP-pep-CR2, ELP-control, pep-CR2 treated and no treatment control group. Images were acquired on an EVOS-FL microscope with $4 \mathrm{X}$ maginification.

\section{Discussion}

In this study, we utilized multiple approaches to demonstrate that CR2 of HCMV tegument protein pp150 is amenable to targeting by sequence specific peptide pep-CR2. We used ELP as a suitable conjugate and carrier system to enhance the bioavailability of pepCR2 for future applications in an in-vivo system. We first screened the inhibitory efficiency of peptides that targeted the conserved regions in pp150. The results indicated that upon treatment with peptides that target CR2 of pp150 (pep-CR2), there is a significant reduction in virus growth in cell culture (Figure 3). To further investigate the dose response of pepCR2 on infected human fibroblasts we performed an $\mathrm{IC}_{50}$ assay using a concentration range of 1 to $20 \mu \mathrm{M}$. The half maximal inhibitory concentration was calculated to be $1.33 \mu \mathrm{M}$ (Figure $4 \mathrm{~A}$ ) and pep-CR2 showed $\sim 90 \%$ inhibition of virus growth at $10 \mu \mathrm{M}$ concentration (Figure 4B). Pep-CR2 treatment of HFFs did not affect cell viability for the duration of treatment (Figure S3A) confirming that the observed reduction in virus titer was not due to cell death. Moreover, when HFs were pretreated and maintained in pep-CR2 throughout the course of infection, the cells resisted infection induced cell death at late time postinfection (Figure S3B). To understand the possible mechanism of inhibition of HCMV replication by pep-CR2, we looked at the cytoplasmic and nuclear localization of pp150 at late time post-infection. The results indicated that pp150 is sequestered in the nucleus of infected cells that are treated with pep-CR2 (Figure 5). This would conform to a mechanistic disruption of pp150 loading onto capsids and subsequent cytoplasmic egress which might be compromising vAC organization and virion maturation process.

Tegument proteins play a critical role in the CMV life cycle process in the host cell and can be considered as important targets for the development of antiviral therapy. Pp150 is a late viral tegument protein which supports the final steps of the maturation process. CMV infection can persist in latent, chronic and productive stages depending on the host physiology and the types of cells being infected. CMV most likely establishes life-long persistence by replicating at a subclinical level and occasionally reactivating from the latent stage triggered by various biological, chemical or physical stimulations including immunological disorders, radiation and cell differentiation among others $[4,39,40]$. Therefore, an effective antiviral against CMV would target cell to cell spread of latently reactivated virus. This can be effectively achieved by targeting late stages of virus maturation.

Although peptides are promising therapeutic strategies, they are easily degraded in-vivo and show poor pharmacokinetic properties as well as cell penetration. To overcome these limitations, we conjugated pep-CR2 with a biopolymer (ELP) as a suitable carrier molecule. 
We successfully designed, expressed in E. coli and purified the ELP-pep-CR2 chimeric fusion protein (Figure S1, S2). Next, we tested the inhibitory potential of ELP-pep-CR2 on HFFs. This showed significant reductions in virus titer upon treatment with ELP-pep-CR2 (Figure 6A), albeit at a lower potency than the free peptide. The reasons for the reduced potency could be related to the relative ability of the free pep-CR2 peptide or the ELP-pepCR2 fusion protein to enter the cells. Future studies will assess cellular uptake to determine if ELP fusion reduces the amount of peptide delivered to the cells. Additionally, if this is the case, we may employ the use of cell penetrating peptides to increase intracellular delivery of the viral inhibitory peptide, a strategy we have successfully utilized for delivery of other peptide therapeutics $[41,42]$. Alternatively, the reduced potency may be due to an altered ability to bind to the pp150 target protein when the CR2 peptide is fused to the large ELP carrier. This can be addressed by modifying the size of the ELP, varying the linker length between the ELP and the CR2 peptide, or by introducing a cleavable linker. Importantly, the reduced in vitro potency does not necessarily predict a lower in vivo efficacy. The fusion of the CR2 peptide to the ELP carrier will likely result in improved in vivo pharmacokinetics and bioavailability, as we have observed with other ELP-delivered peptides [43]. Therefore, pharmacological advantages of ELP fusion could offset the reduction in potency. Interestingly, the ELP-control treated group also showed some non-specific inhibition of virus growth (Figure 6B). The calculated IC $_{50}$ of ELP-control was significantly higher compared to ELP-pep-CR2, and virus inhibition was observed only at higher concentration treatments. The ELP control treated group produced significantly larger sized plaques compared to smaller plaques in the ELP-pep-CR2 treated group (Figure 7A, 7B). The smaller sized plaques indicate reduction in cell-to-cell virus spread in a foci, inferring reduction in virus growth. The mechanism for this non-specific inhibition by the ELP control is unknown, but may be related to effects of large doses of ELP on cellular endocytosis/exocytosis processes. Treatment with ELP or ELP-pep-CR2 did not affect cell viability at the treated concentration during the whole experimental timeline, indicating that neither ELP or the ELP peptide conjugate is cytotoxic (Figure. S4A). Similar to pep-CR2, the conjugated ELP-pep-CR2 treatment also protected infected cells against virus induced lytic cell death (Figure S4B). These experiments indicate that ELP-pep-CR2 show similar inhibitory potential compared to pep-CR2 in HCMV infected cells. The antiviral efficacy of ELPpep-CR2 vs. pep-CR2 will be followed up in a mouse model of CMV infection and later in a clinical trial. Overall, the results in this study show that CR2 of pp150 is amenable to targeting by sequence specific peptide inhibitor which can be developed into an effective antiviral.

Supplementary Materials: The following are available online at www.mdpi.com/xxx/s1, Figure S1, S2, S3 and S4.

Author Contributions: RT and GLB designed and guided the experiments; DM, MHH, GLB and RT performed the experiments and analyzed the data. RT and DM wrote and edited the manuscript.

Conceptualization, R.T. , D.M., and G.L.B.; methodology, R.T, MHH, D.M., and G.L.B.; software, D.M.; validation, R.T. , D.M., and G.L.B.; formal analysis, R.T. , D.M., and 
G.L.B.; investigation, R.T. , D.M., and G.L.B.; resources, R.T. , and G.L.B.; writing - original draft preparation, D.M.; writing - review and editing, R.T. and G.L.B.; supervision, R.T.; project administration, R.T.; funding acquisition, R.T. All authors have read and agreed to the published version of the manuscript.

Funding: This research was funded by NASA, grant number 80NSSC19K1603 (PI: Tandon).

Data Availability Statement: All data is included in the manuscript itself.

Acknowledgments: The authors thank Rowshan Begum, Adesanya Akinleye, and John A. Howell for assistance with polypeptide purification, Lauren A. Fassero for assistance with cell culture maintenance, and John T. Bates for providing help with the PyMOL software.

Conflicts of Interest: G.L.B. is an inventor on patents related to ELP delivered therapeutics and is owner of Leflore Technologies, a private company working to develop ELP drug delivery technology. 


\section{References}

1. Britt, W. Manifestations of human cytomegalovirus infection: proposed mechanisms of acute and chronic disease. Curr Top Microbiol Immunol 2008, 325, 417-470, doi:10.1007/978-3-540-77349-8_23.

2. Griffiths, P.; Reeves, M. Pathogenesis of human cytomegalovirus in the immunocompromised host. Nat Rev Microbiol 2021, doi:10.1038/s41579-021-00582-z.

3. Britt, W.J. Congenital Human Cytomegalovirus Infection and the Enigma of Maternal Immunity. J Virol 2017, 91, doi:10.1128/JVI.02392-16.

4. Mocarski, E.S., Jr., Shenk, T., Pass R. F. Cytomegaloviruses., p. 2701-2772. In D. M. Knipe and P. M. Howley (ed.), Fields Virology. 5th Edition. Lippincott Williams \& Wilkins, Philadelphia. 2006.

5. Mettenleiter, T.C.; Klupp, B.G.; Granzow, H. Herpesvirus assembly: a tale of two membranes. Curr Opin Microbiol 2006, 9, 423-429, doi:10.1016/j.mib.2006.06.013.

6. Hellberg, T.; Passvogel, L.; Schulz, K.S.; Klupp, B.G.; Mettenleiter, T.C. Nuclear Egress of Herpesviruses: The Prototypic Vesicular Nucleocytoplasmic Transport. Adv Virus Res 2016, 94, 81-140, doi:10.1016/bs.aivir.2015.10.002.

7. Alwine, J.C. The human cytomegalovirus assembly compartment: a masterpiece of viral manipulation of cellular processes that facilitates assembly and egress. PLoS Pathog 2012, 8, e1002878, doi:10.1371/journal.ppat.1002878.

8. Procter, D.J.; Banerjee, A.; Nukui, M.; Kruse, K.; Gaponenko, V.; Murphy, E.A.; Komarova, Y.; Walsh, D. The HCMV Assembly Compartment Is a Dynamic Golgi-Derived MTOC that Controls Nuclear Rotation and Virus Spread. Dev Cell 2018, 45, 83-100 e107, doi:10.1016/j.devcel.2018.03.010.

9. Tandon, R.; Mocarski, E.S. Viral and host control of cytomegalovirus maturation. Trends Microbiol 2012, 20, 392-401, doi:10.1016/j.tim.2012.04.008.

10. Britt, B. Maturation and egress. In Human Herpesviruses: Biology, Therapy, and Immunoprophylaxis, Arvin, A., Campadelli-Fiume, G., Mocarski, E., Moore, P.S., Roizman, B., Whitley, R., Yamanishi, K., Eds.; Cambridge, 2007.

11. Kalejta, R.F. Tegument proteins of human cytomegalovirus. Microbiol Mol Biol Rev 2008, 72, 249-265, table of contents, doi:10.1128/MMBR.00040-07.

12. Mocarski, E.S., Jr.; Shenk, T.; Pass, R.F. Fields Virology, 5th ed.; Howley, D.M.K.P.M., Ed.; Lippincott Williams \& Wilkins: Philadelphia, PA, USA, 2006.

13. AuCoin, D.P.; Smith, G.B.; Meiering, C.D.; Mocarski, E.S. Betaherpesvirus-conserved cytomegalovirus tegument protein ppUL32 (pp150) controls cytoplasmic events during virion maturation. J Virol 2006, 80, 8199-8210, doi:10.1128/JVI.00457-06.

14. Tandon, R.; Mocarski, E.S. Control of cytoplasmic maturation events by cytomegalovirus tegument protein pp150. J Virol 2008, 82, 9433-9444, doi:10.1128/JVI.00533-08.

15. Baxter, M.K.; Gibson, W. Cytomegalovirus basic phosphoprotein (pUL32) binds to capsids in vitro through its amino one-third. J Virol 2001, 75, 6865-6873, doi:10.1128/JVI.75.15.6865-6873.2001.

16. Tandon, R.; Mocarski, E.S. Cytomegalovirus pUL96 is critical for the stability of pp150-associated nucleocapsids. J Virol 2011, 85, 7129-7141, doi:10.1128/JVI.02549-10.

17. Brechtel, T.M.; Mocarski, E.S.; Tandon, R. Highly acidic C-terminal region of cytomegalovirus pUL96 determines its functions during virus maturation independently of a direct pp150 interaction. J Virol 2014, 88, 4493-4503, doi:10.1128/JVI.03784-13. 
18. Liu, W.; Dai, X.; Jih, J.; Chan, K.; Trang, P.; Yu, X.; Balogun, R.; Mei, Y.; Liu, F.; Zhou, Z.H. Atomic structures and deletion mutant reveal different capsid-binding patterns and functional significance of tegument protein pp150 in murine and human cytomegaloviruses with implications for therapeutic development. PLoS Pathog 2019, 15, e1007615, doi:10.1371/journal.ppat.1007615.

19. Bogdanow, B.; Weisbach, H.; von Einem, J.; Straschewski, S.; Voigt, S.; Winkler, M.; Hagemeier, C.; Wiebusch, L. Human cytomegalovirus tegument protein pp150 acts as a cyclin A2-CDK-dependent sensor of the host cell cycle and differentiation state. Proc Natl Acad Sci U S A 2013, 110, 17510-17515, doi:10.1073/pnas.1312235110.

20. Dai, X.; Yu, X.; Gong, H.; Jiang, X.; Abenes, G.; Liu, H.; Shivakoti, S.; Britt, W.J.; Zhu, H.; Liu, F.; et al. The smallest capsid protein mediates binding of the essential tegument protein pp150 to stabilize DNA-containing capsids in human cytomegalovirus. PLoS Pathog 2013, 9, e1003525, doi:10.1371/journal.ppat.1003525.

21. Indran, S.V.; Ballestas, M.E.; Britt, W.J. Bicaudal D1-dependent trafficking of human cytomegalovirus tegument protein pp150 in virus-infected cells. J Virol 2010, 84, 3162-3177, doi:10.1128/JVI.01776-09.

22. Moorman, N.J.; Sharon-Friling, R.; Shenk, T.; Cristea, I.M. A targeted spatial-temporal proteomics approach implicates multiple cellular trafficking pathways in human cytomegalovirus virion maturation. Mol Cell Proteomics 2010, 9, 851-860, doi:10.1074/mcp.M900485-MCP200.

23. Sampaio, K.L.; Cavignac, Y.; Stierhof, Y.D.; Sinzger, C. Human cytomegalovirus labeled with green fluorescent protein for live analysis of intracellular particle movements. J Virol 2005, 79, 2754-2767, doi:10.1128/JVI.79.5.2754-2767.2005.

24. Yu, X.; Jih, J.; Jiang, J.; Zhou, Z.H. Atomic structure of the human cytomegalovirus capsid with its securing tegument layer of pp150. Science 2017, 356, doi:10.1126/science.aam6892.

25. Yu, X.; Shah, S.; Lee, M.; Dai, W.; Lo, P.; Britt, W.; Zhu, H.; Liu, F.; Zhou, Z.H. Biochemical and structural characterization of the capsid-bound tegument proteins of human cytomegalovirus. J Struct Biol 2011, 174, 451-460, doi:10.1016/j.jsb.2011.03.006.

26. Bidwell, G.L., 3rd; Raucher, D. Cell penetrating elastin-like polypeptides for therapeutic peptide delivery. Adv Drug Deliv Rev 2010, 62, 1486-1496, doi:10.1016/j.addr.2010.05.003.

27. Lee, A.C.; Harris, J.L.; Khanna, K.K.; Hong, J.H. A Comprehensive Review on Current Advances in Peptide Drug Development and Design. Int J Mol Sci 2019, 20, doi:10.3390/ijms20102383.

28. Di, L. Strategic approaches to optimizing peptide ADME properties. AAPS J 2015, 17, 134-143, doi:10.1208/s12248-014-9687-3.

29. Urry, D.W. Free energy transduction in polypeptides and proteins based on inverse temperature transitions. Prog Biophys Mol Biol 1992, 57, 23-57, doi:10.1016/0079-6107(92)90003-o.

30. Arias, F.J.; Reboto, V.; Martin, S.; Lopez, I.; Rodriguez-Cabello, J.C. Tailored recombinant elastin-like polymers for advanced biomedical and nano(bio)technological applications. Biotechnol Lett 2006, 28, 687-695, doi:10.1007/s10529-006-9045-3.

31. Bidwell, G.L., 3rd; Fokt, I.; Priebe, W.; Raucher, D. Development of elastin-like polypeptide for thermally targeted delivery of doxorubicin. Biochem Pharmacol 2007, 73, 620-631, doi:10.1016/j.bcp.2006.10.028.

32. Chilkoti, A.; Dreher, M.R.; Meyer, D.E.; Raucher, D. Targeted drug delivery by thermally responsive polymers. Adv Drug Deliv Rev 2002, 54, 613-630, doi:10.1016/s0169-409x(02)00041-8.

33. Lee, J.; Macosko, C.W.; Urry, D.W. Elastomeric polypentapeptides cross-linked into matrixes and fibers. Biomacromolecules 2001, 2, 170-179, doi:10.1021/bm0000900.

34. MacEwan, S.R.; Chilkoti, A. Applications of elastin-like polypeptides in drug delivery. J Control Release 2014, 190, 314-330, doi:10.1016/j.jconrel.2014.06.028. 
35. Nowatzki, P.J.; Tirrell, D.A. Physical properties of artificial extracellular matrix protein films prepared by isocyanate crosslinking. Biomaterials 2004, 25, 1261-1267, doi:10.1016/s0142-9612(03)00635-5.

36. Srokowski, E.M.; Woodhouse, K.A. Development and characterisation of novel cross-linked bio-elastomeric materials. J Biomater Sci Polym Ed 2008, 19, 785-799, doi:10.1163/156856208784522038.

37. George, E.M.; Liu, H.; Robinson, G.G.; Mahdi, F.; Perkins, E.; Bidwell, G.L., 3rd. Growth factor purification and delivery systems (PADS) for therapeutic angiogenesis. Vasc Cell 2015, 7, 1, doi:10.1186/s13221-014-0026-3.

38. Mierendorf, R.C.; Morris, B.B.; Hammer, B.; Novy, R.E. Expression and Purification of Recombinant Proteins Using the pET System. Methods Mol Med 1998, 13, 257-292, doi:10.1385/0-89603-485-2:257.

39. Mehta, S.K.; Laudenslager, M.L.; Stowe, R.P.; Crucian, B.E.; Sams, C.F.; Pierson, D.L. Multiple latent viruses reactivate in astronauts during Space Shuttle missions. Brain Behav Immun 2014, 41, 210-217, doi:10.1016/j.bbi.2014.05.014.

40. Goodrum, F. Human Cytomegalovirus Latency: Approaching the Gordian Knot. Annu Rev Virol 2016, 3, 333 357, doi:10.1146/annurev-virology-110615-042422.

41. Bidwell, G.L., 3rd; Davis, A.N.; Raucher, D. Targeting a c-Myc inhibitory polypeptide to specific intracellular compartments using cell penetrating peptides. J Control Release 2009, 135, 2-10, doi:10.1016/j.jconrel.2008.11.015.

42. Massodi, I.; Bidwell, G.L., 3rd; Raucher, D. Evaluation of cell penetrating peptides fused to elastin-like polypeptide for drug delivery. J Control Release 2005, 108, 396-408, doi:10.1016/j.jconrel.2005.08.007.

43. Eddy, A.C.; Howell, J.A.; Chapman, H.; Taylor, E.; Mahdi, F.; George, E.M.; Bidwell, G.L., 3rd. BiopolymerDelivered, Maternally Sequestered NF-kappaB (Nuclear Factor-kappaB) Inhibitory Peptide for Treatment of Preeclampsia. Hypertension 2020, 75, 193-201, doi:10.1161/HYPERTENSIONAHA.119.13368. 